\title{
The Shear Forming Mechanism Research of Matrix Arrangement Cement Lining Steel
}

\author{
ZHANG Min ${ }^{1, a}$, GUO Shuai ${ }^{2, b}$ and ZOU Shu-liang ${ }^{3, c}$ \\ ${ }^{1,3}$ School of Nuclear Science and Technology, University of South China, HengYang 421001,China \\ ${ }^{1}$ School of Mathematics and Physics Science,University of South China,HengYang, 421001,China \\ ${ }^{1,3}$ Hunan Provincial Key Laboratory of Emergency Safety Technology and Equipment for Nuclear \\ Facilities ,HengYang 421001,China \\ ${ }^{2}$ CRUN Power Equipment Co., Ltd, ZiGong, 643000,China \\ a emin1217@sina.com, bjiurenkaihei@sina.cn, 'zoushuliang@yahoo.com.cn
}

Keywords: Cement lining steel pipe; Rigid-flexible coupling; Plastic yield; Mathematical model

\begin{abstract}
It is because the matrix arrangement cement lining steel shear which have large elastic-plsatic deformation,yield deformation and slip body ,as well as existing multiple complex nonlinear and coupling that traditional methods have not been able to meet the analysis requirements. Therefore, The coupled theory based on elastic mechanics, fracture mechanics and brittle fracture was put forward by considering the change"elastic deformation-plastic yield-shear fracture", and having in-depth discussion about the sheear process.Eventually,a complete mathematical model of matrix arrangement cement lining steel shear has been formed to provides theoretical basis for shear performance and mechanics analysis.
\end{abstract}

\section{Introduction}

The cement lining steel pipe is formed by daubing cement mortar in the wall of pipe,and then pour into concrete,so it has certain flexibility,as well as can absorb a lot of heat.because of unique structure composition and good use value that the cement lining steel pipe has caused the wide attention of researchers.It is a complex large elastic-plastic deformation process to Shear cement lining steel pipe,and there not only exists physical nonlinear relation between stress and train,but also has geometric nonlinear relationship between displacement and strain.In this paper,a mathematical model was established to simulate the shear of multiple tube from just yielding fracture and steel cement pipe between the brittle fracture of mutual influence ${ }^{[1]}$.

\section{The Shear Principle of Concrete Lining of Steel Pipe}

In the shear process, the steel tube was fixtured into the cropping die, through the two phase fault tools before and after to shear, the power system of shearing machine was used to promote the main cutting tool move forward, and make the steel tube occur elastic-plastic deformation and rupture, while cement tube directly appear rigid brittle fracture under the action of shear force ${ }^{[2]}$, as shown in figure 1.

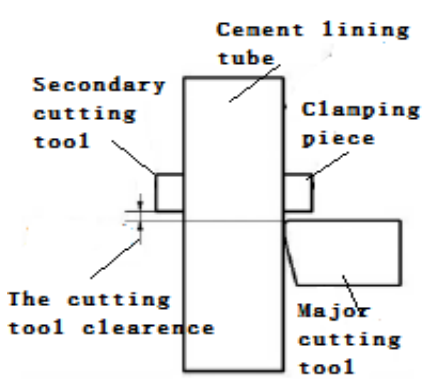

Fig1.The Shear principle 


\section{The Matrix Arrangement Model of Cement Lining Steel Pipe}

The matrix cement lining steel pipe's model as a permutation and combination with 5 row 5 column,and steel pipe diameter is $5 \mathrm{~mm}$,wall thickness is $0.65 \mathrm{~mm}$,inside evenly filled with solid cement.The cement pipe's top was constrained,so the shear machine start to cut where has a certain distance from the bottom ,its shear device uses a combination that three cutter arrange ladder shaped,and cutting tool material was steel bonded carbide GW30.The components was constrainted by either side of pipe clamp devices,the cutting tool shear tube at speed of $0.001 \mathrm{~m} / \mathrm{s}$, then all the cut pipe was set into the lower recovery unit, the cutter model, shear model and matrix arrangement of tubes are shown in figure 2 .
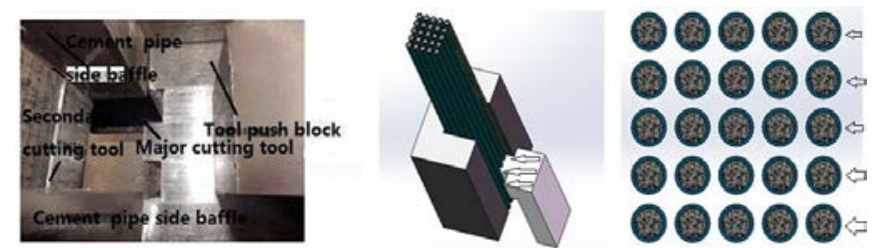

Fig2 The shear model of matrix lining concrete steel tube

\section{The Rigid-flexible Coupling Mathematical Model of Shearing Matrix Cement Lining Steel}

\section{The Teel Tube Shear Theory}

Since the Cosserat brothers introduct the bending moment intoinfinitesimal body balance, first proposed the Cosserat shear theory, on this basis, the nonlocal theory is put forward that has very good guidance function for studying shearing area, it can be a very good mathematical model to describe the ductile fracture in the process of material shear fracture ${ }^{[3]}$.Eventually nonlocal concept was put forward by Eringen, and expression as follows.

$$
\sigma=E \bar{\varepsilon}
$$

Among them, the nonlocal strain is shown in(2-2)

$$
\bar{\varepsilon}(x)=\frac{1}{V} \int_{V} h(s) \varepsilon(x+s) d V
$$

Based on the coupling stress theory, a pair of shearing force can be described in the Cauchy stress:

$$
\tau_{i j}=\sigma_{i j}+r_{i j}
$$

$\sigma \mathrm{ij}$ and $\tau \mathrm{ij}$ is symmetrical part,while rij and $\tau \mathrm{ij}$ is antisymmetric parts.

Also the deformation geometry equations and the balance equation as follows.

$$
\varepsilon_{i j}=\frac{1}{2}\left(u_{i j}+u_{j_{i}}\right) \quad m_{i j}+e_{i s t} \tau_{i j}=0
$$

According to the yield limit of the virtual work principle

$$
\int_{V}\left(\sigma_{i j} \delta \varepsilon_{i j}+m_{i j} \delta \lambda i_{i j}\right) d V=\int_{S}\left(t_{i} u_{i}+w_{i} q_{i}\right) d S
$$

The shear deformation constitutive equation is shown (2-9)

$$
\sigma_{i j}=E_{i j k} \varepsilon_{i j}
$$

Whereby, ${ }^{M_{i k l}}=2 G^{2} \delta_{i j} \delta_{j i}$ is a high order model of the corresponding shear stress ${ }^{[4]}$. 
For the elastomer's field function"f", plastic yielding must meet"g = f", its necessary conditions is plastic consistency

$$
\frac{\partial f}{\partial \sigma_{i j}} \dot{\sigma}_{i j}+\frac{\partial f}{\partial m_{i j}} \dot{m}_{i j}+\frac{\partial f}{\partial \lambda} \dot{\lambda}=0
$$

From (2-4) (2-7) can get the strength stress formula of steel pipe ,shown in (2-8)

$$
\dot{\sigma}_{i j}=E_{i j k l}^{e p} \dot{\varepsilon}_{i j}-E_{i j k l}^{c} \dot{\lambda}_{i j} \quad \dot{m}_{i j}=M_{i j k l}^{e p} \dot{\varepsilon}_{i j}-M_{i j k l}^{c} \dot{\lambda}_{i j}
$$

So the limit of yielding fracture conditions for steel pipe was as follows.

$$
\sigma_{i j}>\left[\sigma_{s}\right] \quad m_{i j}>\left[\sigma_{0.2}\right]
$$

\section{The Cement Pipe Fracture Theory}

In order to explain the difference between theoretical strength and actual strength of glass, Griffith put forward the microcrack theory,and it gradually developed into the core of brittle fracture.Griffith think brittle material always exist tiny crack or flaw, the stress concentration phenomenon was appeared under the action of external force,when the stress reaches a certain degree, crack began to expand, eventually lead to fracture ${ }^{[5]}$.

From the energy balance standpoint,Griffith deduce the conditions of crack propagation.

$$
\sigma_{C}=\sqrt{\frac{2 E \lambda}{\left(1-\mu^{2}\right) \pi c}}
$$

While,the theory combined strength material is connected with how to control the crack length and atomic spacing orders of magnitude

$$
\sigma_{C}=\sqrt{\frac{E \lambda}{4 c}}
$$

\section{The Rigid-flexible Coupling Theory of Cement Lining Tube}

The process of shearing cement lining steel pipe belongs to typical rigid-flexible coupling model , yield rupture of steel tube is a specific attribute of flexible body, and rigid brittle fracture is natural property of rigid body.because cement pipe increased the rigidity of steel pipe ,In the beginning stages , steel pipes' stress transfer must through ring to pass by edge, only the middle is blocked and intermittent by cement block, so that make the steel pipe has good shearing area, also the extrusion produce certain preloading in advance, to a great extent, promote the cement rod rigid brittle fracture.In order to analyze conveniently, regard"r"as flexible steel,and "f" as rigidity, according to the research conclusion of Shanghai jiaotong university's project group,by using Jourdain variational principle and Lagrange equation, the dynamic equations's representation are shown as follows.

$$
\left[\begin{array}{l}
u_{r} \\
u_{q}
\end{array}\right]=\left[\begin{array}{c}
w_{r}-y \frac{\partial w_{q}}{\partial x}-\int_{0}^{x} \frac{1}{2}\left[\left(\frac{d w_{q}}{\partial x}\right)^{2}\right] d \xi \\
w_{q}
\end{array}\right]
$$

After the finite element method was used to discrete, as follows.

$$
\left[\begin{array}{l}
u_{r} \\
u_{q}
\end{array}\right]=\left[\begin{array}{c}
N_{r} p-y \frac{\partial N_{q}}{\partial x} p-\frac{1}{2} p^{T} H p \\
N_{q} p
\end{array}\right]
$$


So,the Coupling function matrix can be obtained.

$$
H=B_{k}^{T} \int_{0}^{\bar{x}} \frac{\partial N_{r}^{K T}}{\partial \bar{x}} \bullet \frac{\partial N_{q}^{K}}{\partial \bar{x}} d \bar{x} B_{k}+\sum_{i=1}^{k=1} B_{i}^{T} \int_{0}^{T_{i}} \frac{\partial N_{r}^{i T}}{\partial \bar{x}} \bullet \frac{\partial N_{q}^{i}}{\partial \bar{x}} d \bar{x} B_{i}
$$

$\left(u_{r}, u_{q}\right)$ is strain of arbitrary point in the cartesian coordinate system, (wr, wq) is deformation of corresponding points.

\section{The Rigid-flexible Coupling Mathematical Model of Shearing Matrix Composite Tube}

The shear process of matrix arrangement cement lining steel pipe need to consider the steel tube plastic fracture,cement pipe rigid tube rupture , and coupled action between multiple tube extrusion.It is considering the stiffness of the concrete lining pipe has increased that the mutual influence between the pipe is mainly manifested on the tool feed direction, just as shown in figure 2.So we can hypothesize Steel pipe permutation matrix is expressed as " $A$ ",cement pipe is expressed as" $\mathrm{B}$ ",interaction between the pipe is expressed as the matrix "Z",attenuation of forces is matrix "is",as well as the coupled effect is "H".So the necessary condition to make cement lining steel shear fracture was shown as follows.

$$
\begin{gathered}
\sigma_{[A]}>\sigma_{s} \quad \sigma_{[B]}>\sigma_{c} \\
\sigma[A]=\left[\sigma a_{x y_{i j}} H\left(1+Z Y a_{x y}\right)\right] \quad(x=1 \sim 5, y=1 \sim 5) \\
\sigma_{[B]}=\frac{1}{1-\chi} \sigma_{[A]}
\end{gathered}
$$

Among them, the principal strain and shear strain expressed by cutting speed,and $\mathrm{v}$ is cutter track speed, $\zeta$ is cutting tool clearance.

$$
\dot{\sigma}_{i j}=E_{i j k l}^{e p} \dot{\varepsilon}_{i j}-E_{i j k l}^{c} \dot{\lambda}_{i j} \quad \dot{\varepsilon}_{i j}=\delta_{t} v \quad \dot{\lambda}_{i j}=\frac{\delta_{t} v}{\varsigma}(1+Z Y)
$$

Eventually,just put (2-22),(2-23),(2-24) into (2-21),so that the rigid-flexible coupling mathematical model of shearing matrix cement lining steel was obtained.

$$
\left\{\begin{array}{c}
{\left[E_{j p k}^{e p} \delta_{t} v-E_{i j k}^{c} \frac{\delta_{t} v}{\varsigma}(1+Z Y)\right] H(1+Z Y)>\left[\sigma_{s}\right]} \\
\frac{1}{1-\chi}\left[E_{i j k}^{e p} \delta_{t} v-E_{i j k l}^{c} \frac{\delta_{t} v}{\varsigma}(1+Z Y)\right] H(1+Z Y)>\sqrt{\frac{2 E \lambda}{\left(1-\mu^{2}\right) \pi c}}
\end{array}\right\}
$$

\section{Conclusion}

The shear model of cement lining steel has very complex multiple nonlinear and coupling, is a ntegrated complex theory that it is involved with elastic mechanics, fracture mechanics, the plastic yield, and coupled .In the process of shearing, so all cutter feeding speed, pipe spacing arrangement, cutting tool ,cutting tool clearance, and tool performance will affect the shear mechanism and formability.This paper aim to establish a mathematical model by integrated all of the above factors,so it can provide reliable theoretical basis for further studying the evaluation of shear performance and the finite element analysis .

\section{References}

[1]FAN Yan-ru,WANG Xiang-jiang,TANG Yong-liang.Numerical Simulation of Metal Bar Shearing[J].MANUFACTURING INFORMATIZATION,2013,6:84-87

[2]M.Mazurkiewicz.A manufatuing tool for a new century.Journal of Materials Porcessing 
Technology,2000,(106):112-118.

[3]SONG Qin-qin,HAO Nan-hai.Different Shear Gap's Finite Element Analysis of Steel Shear[J].Machine Building\&Automation.2012,7(4):47-50

[4]Yang F,Chong ACM,Lam DCC,etal.Couple stress based strain gradient theory for elasticity. International Journal of Solids and Structures,2002,39(10):2731-2743

[5]Zhang L.A separated law of hardening in strain gradient plasticity.Acta Mechanica Sinica, 1997,13(2):161-164 